\title{
The Effect of Sunscreens on Yeast to Prevent Ultraviolet Damage
}

\author{
Tomather Alhamdy¹, Noorah Saleh Al-Sowayan ${ }^{2 *}$ \\ ${ }^{1}$ Department of Biology, College of Science, Qassim University, Buraydah, KSA \\ ${ }^{2}$ Department of Biology, Faculty of Science, Qassim University, Buraydah, KSA \\ Email: ^nsaoiean@qu.edu.sa, *knaaj1@yahoo.com
}

How to cite this paper: Alhamdy, T. and Al-Sowayan, N.S. (2020) The Effect of Sunscreens on Yeast to Prevent Ultraviolet Damage. Advances in Bioscience and Biotechnology, 11, 111-122.

https://doi.org/10.4236/abb.2020.114009

Received: March 10, 2020

Accepted: April 19, 2020

Published: April 22, 2020

Copyright $\odot 2020$ by author(s) and Scientific Research Publishing Inc. This work is licensed under the Creative Commons Attribution International License (CC BY 4.0).

http://creativecommons.org/licenses/by/4.0/

\begin{abstract}
The impacts of Ultraviolet (UV) radiation on the human body have received increased attention in previous years. The impacts are comprised of a series of illnesses, burns, and modifications in the genetic structures. Therefore, the current study aimed to identify the effect of sunscreens on yeast to prevent ultraviolet damage. The study uses two sunscreens with different sun protection factors to show how they differently protect the cells from the wrath of UV light. The experiment involves the use of yeast as the test organism since yeast has DNA that is used for reproduction by budding. UV light affects the growth of the bacteria and the limiting factor with the sunscreens. Six Petri dishes with agar were inoculated with yeast solution and put under different conditions and the growth results will be obtained. The experiment also showed how different clothes protect human from the UV radiations.
\end{abstract}

\section{Keywords}

Sunscreen, Ultraviolet Damage, Ultraviolet Radiations, Yeast

\section{Introduction}

The sun emits long wavelength radiation in form of visible and invisible light. The invisible light houses the ultra violet light which can be classified in three types: UVA, UVB and UVC. UVA is not absorbed by the ozone layer and therefore it directly comes to the troposphere; it penetrates on the skin cells causing premature aging of the skin and affects the immunity. UVD is absorbed partially by ozone its responsible of DNA damage which leads to squamous cell carcinoma as well cell as cell apoptosis evidenced by the peeling of the skin after UV exposure. UVC is completely absorbed by the ozone layer.

The negative impacts of these radiations can be minimised by using sun- 
screens which may further be categorised into organic and inorganic materials. However, it is also of equal importance that people must be aware of the protection provided by these sunscreens [1]. Based on materials being used, these can be broadly divided into two major categories including organic and non-organic sunscreens. For assessing the effectiveness of sunscreens against ultraviolet radiation, sun protection factor (SPF) is widely used as a factor [2]. The ease in culturing Saccharomyces cerevisiae, a yeast, makes it an optimal choice for scientists to use it as experiment material for identifying the impact of UV irradiation-caused damage in DNA [1]. The other rationale for its extensive use in the research process is based on similarities in reproduction, metabolism, and chemistry of Saccharomyces cerevisiae and human cells. The use of Saccharomyces cerevisiae has been used historically to identify the identical impacts of ultraviolet radiation on the human's skin cells because it has the same DNA for repair as humans. They therefore show the same metabolic change when exposed to UV light. When it is exposed to UV, yeast gets mutations make them impossible to perform DNA repair leading to the death of yeast cells.

The problem statement of the current study is to assess the impact of ultraviolet radiation on the yeast. Precisely, these results will outline the usefulness of the available sunscreen in preventing ultraviolet damages. The effects on yeast will be assessed for all types of ultraviolet radiation for making the assessment comprehensive in nature. The major effect which will be analysed in the current study is the effect of sunscreens on the colonial growth of yeast as it outlines the growth and development of yeast cells in the presence and absence of ultraviolet radiation.

The main objective of the study is to identify the effect of sunscreens on yeast to prevent damage which is caused by UV radiation exposure and therefore get the exact effect of sunscreen on human beings in preventive UV radiation toxicity.

\subsection{Abilities of Sunscreens to Screen Ultraviolet Radiations}

$\mathrm{Xu}$ and Liu [3] outlined that the use of yeast in researches concerning the impact of ultraviolet radiation is not new to the researchers as these have been used extensively in the researches. They also claimed that ease in culturing yeasts makes an optimal choice for the researchers who design their researchers by using yeast to identify the efficacy of the sunscreen as well. They also argued that photoprotection is important as ultraviolet radiations continue to develop a series of skin issues which may continue to increase with an increase in the exposure to ultraviolet radiation. Rosenthal and Burchum [4] outlined that photoprotection is an increasing concern among the common public who may continue to rely on a series of sunscreen considering that these screens will be able to serve the purpose. Sudhahar \& Balasubramanian [5] conducted a comprehensive study for identifying the impacts of sunscreens on microbial. They outlined that the impact of each of sunscreen is different as each of the available sunscreens have their own effectiveness. They outlined that some of the sunscreens like zinc oxide 
may be able to absorb UVA and UVB radiations effectively while UV screening capacity of others may not be too high to protect the skin effectively.

Green et al., [6] conducted a comprehensive study for identifying the efficacy of different sunscreen including SPF $>15$. They argued that the SPF level of more than 15 is selected as it is considered as the highest level of safety in terms of screening UVA and UVB. They argued that using sunscreens may be helpful in avoiding a series of healthcare issues like Invasive melanoma. They, however, pointed out that using sunscreens regularly may result in better outcomes rather than using it occasionally. Ghiasvand et al., [7] also identified that using sunscreens having SPF higher than 15 may be helpful for the people in avoiding the negative impacts of ultraviolet radiation. Stiefel et al., [8] also outlined the sunscreens having SPF lower than 15 may not be considered as appropriate and sufficient for avoiding the impacts of ultraviolet radiations fully as the ability to screen ultraviolet radiations is limited in the case of such screens even applied regularly. Waldman \& Grant-Kels [9] conducted a comprehensive study for identifying the efficacy of sunscreens in helping users avoiding serious healthcare issues. They found that using sunscreen having SPF higher than 15 is helpful in avoiding serious healthcare issues like melanoma and non-melanoma cancers which may, otherwise, be a case due to direct exposure of the skin to the sun. Shaath [10] argued that the use of sunscreens does not have straightforward outcomes rather some of the sunscreens may fail to protect the skins from such effects due to their lower SPF. They also claimed that occasional application or thin application of sunscreens including all types of sunscreens may not be helpful in avoiding such serious health outcomes.

On the other hand, Kowalski [11] outlined that using sunscreen may be helpful up to some extent only and may not be considered as an optimal solution. $\mathrm{He}$ argued that sunscreen, irrespective of SPF, fails to stop all of the ultraviolet radiation rather these sunscreens are able to filter fewer of them which results in better but not the ultimate protection of skin. Goodman and Fuller [12] have also outlined that sunscreens are only able to filter some ultraviolet radiation and are effective in reducing the intensity of these radiations which may be helpful in reducing the negative impacts on human skin. They pointed out that sunscreens may not be considered as an optimal solution with an ability to complete filtration of ultraviolet radiation. They also outlined that applying thick layers of sunscreen may be helpful up to some extent in reducing the negative impacts significantly; however, complete filtration of ultraviolet radiation is not the case in even this case. Jansen et al., [13] outlined that higher sensitivity of yeast cells is one of the major features which makes it an indicator for the testing processes of sunscreen. They also pointed out that changes in the taxonomy of the sunscreens may change the impact of these solutions on the overall colonial growth of yeast which may indicate a lower SPF.

Hacker et al., [14] conducted a comprehensive study for identifying the efficacy of sunscreens in avoiding damages due to ultraviolet radiation. They outlined that the sunscreens significantly reducing the penetration of ultraviolet 
radiations, however, these do not prevent all of the ultraviolet radiation. They pointed out that some of the factors like sweetening may also impact the performance of the factors which may be followed by a need to reapply the sunscreens. Mazzarello et al., [15] outlined that sunscreens like sunblock sticks are helpful in reducing major skin illnesses. They claimed that some of the serious illnesses like Herpes labialis (HL) recurrences may be reduced significantly due to lower infiltration of ultraviolet radiation. They, however, asserted that the application of sunblock may not be assumed to be a complete solution as they argued that there was a decrease in the overall prevalence of Herpes labialis (HL) recurrences but it does not mean that there was no contact between the ultraviolet radiations and human skill. They asserted that the decrease is associated with a decrease in the ultraviolet radiation passing through the sunscreens. Petersen \& Wulf [16] also believed that using sunscreens may be helpful up to a limit in avoiding the negative impacts of the UV rays; however, it may depend upon SPF and thickness of the layer applied.

\subsection{Sunscreens and Yeast Colonies}

Yeast is used as a testing tool is aimed to identify the ability of a sunscreen to reduce the number of radiations significantly which can pass through the filter [17]. He outlined that extensive exposure of the yeast cells to UV radiations is followed by a decrease in the colonial growth of the yeast cells. They pointed out that the higher rate of colonial growth indicates that the sunscreen remained successful in reducing the ultraviolet radiation below an acceptable level. Sunscreens decrease the exposure of the yeast cells to the ultraviolet radiations which makes the environment favourable for growth [2]. They outlined that the higher growth of yeast assay indicates the lower infiltration of ultraviolet radiation which is one of the desirable outcomes of a sunscreen. Sunscreens are assumed to avoid exposure to ultraviolet radiation which is aimed to minimise the impacts of these radiations [18]. They also outlined that, on the other hand, yeast's colonial growth is highly sensitive to the changes in the environment specifically to an increase in the ultraviolet radiation in the environment. They also believed that yeast tests, therefore, observe the colonial growth of yeast for drawing a conclusion about the efficacy of the sunscreens. The impacts of Bisphenol A and Nonylphenol, are as some sunscreens, on the transcriptional profiles of Saccharomyces cerevisiae Genome [19]. They found that an increase in SPF may be followed by increased growth of Saccharomyces cerevisiae colonies and vice versa which indicates the effectiveness and efficacy of Bisphenol A and Nonylphenol as some of the sunscreens. The growth of yeast colonies is very sensitive to changes in the prevalence of ultraviolet radiation in the environment [20]. They also outlined that the higher rate of growth of yeast assay indicates the lower infiltration of ultraviolet radiation which is one of the desirable outcomes of a sunscreen.

A study conducted for verifying the efficacy of ethyl acetate fraction of Zan- 
thoxylum rhetsa bark extract for identifying its usefulness and efficacy in minimising UVA/UVB radiations [21]. They outlined that SPF must be higher than 15 for making an optimal solution for the testing using yeast. They also outlined that the ability of a sunscreen to minimise the inflow of ultraviolet radiation is the core criteria for making it a successful tool. The yeast colonial growth of yeast is highly associated with the survivability of yeast cells in the presence of ultraviolet radiation [2]. They continued claiming that the use of yeast cells as a tool for carrying out testing is based on the sensitiveness of yeast cells to these radiations. They asserted that the survival of the yeast does not necessarily mean an absence of such factors rather it must be interpreted as a lower amount of the ultraviolet radiation. The role of yeasts in the manufacturing of sunscreens and pointed out that using the yeast as a testing specie is not the sole role of the yeasts rather some of the aquatic yeasts may not only even survive in the presence of high UV but also may use their secretions for avoiding UV filtration [22]. They asserted that it may be helpful in using yeast in the biological sunscreens for increasing the efficacy of sunscreens.

The increased growth of the yeast test indicates that UV radiation is less permeable, which is one of the ideal results for sunscreens [2]. Smaoui et al. [17] described that sunscreens are assumed to avoid exposure to ultraviolet radiation and are designed to minimize the effects of these radiations. They also stressed that the growth of yeast colonies is very sensitive to changes in the environment, in particular the changes in the prevalence of ultraviolet radiation in the environment. They also believe that a yeast test can observe the growth of yeast colonies and draw conclusions about the effectiveness of sunscreens. Kumar [21] described that the use of yeasts in research for identifying the effects of ultraviolet radiation is not new to researchers since these yeasts have been widely used in research preciously as well. They also claimed that the easy-to-grow nature of yeast makes it the best option for researchers.

Most of the yeast colonies are assumed to be sensitive to UV due to their lower survivability, however, there are some yeasts that may not only survive but also work as sunscreens [1] [22]. They also outlined that using yeast as a testing tool may be helpful in determining SPF level of the sunscreen as sunscreen having SPF higher than 15 may facilitate the colonial growth of yeast cell while sunscreen having SPF lower than 15 may fail to protect yeast colonies from ultraviolet radiations followed by lower colonial growth of yeast cells. Marrot [23] analysed different aspects of yeast growth regarding their applicability in pre-testing of sunscreen. They outlined that using yeast for retesting purposes is significant in nature as it reliably determines the level of yeast growth which in the case of lower SPF may be followed by lower colonial growth of yeast. They pointed out the use of yeast in pretesting of sunscreens is not new to the industry rather it has been heavily used for this purpose for a couple of decades. Kim \& Choi [24] outlined that higher sensitivity of yeast cells is one of the major features which makes it an indicator for the testing processes of sunscreen. They also outlined 
that yeasts continue to show higher growth in the case of lower exposure to ultraviolet radiation.

The review of previous literature highlights that the previous studies have involved different aspects regarding the use of yeast in pretesting sunscreens [2] [25] [26]. It is also found that sunscreens are assumed to avoid exposure to ultraviolet radiation and are designed to minimize the effects of these radiations. The literature highlighted that ease in culturing yeasts makes an optimal choice for the researchers who design their researchers by using yeast to identify the efficacy of the sunscreen as well. It is also found that higher sensitivity of yeast cells is one of the major features which make it an indicator for the testing processes of sunscreen. It is also found that extensive exposure of the yeast cells to UV radiations is followed by a decrease in the colonial growth of the yeast cells. The literature highlighted that the higher rate of colonial growth indicates the success of sunscreen broadly. It is also important to consider that the literature, however, lacks quantitative studies for quantifying the impacts in terms of a number of yeast colonies which may require fresh research. This literature gap will be used by the current study as it is quite consistent with the research objectives and questions of the current study.

\section{Materials and Methods}

Materials needed for the experiment are the normal baker's yeast, sugar, nylon cloth, polyester cloth, warm water, and six petri dishes with agar, incubator set at 30 degrees Celsius, pipette, two sunscreens with SPF15, and 50, respectively.

The procedure starts by creating a yeast solution by mixing a small amount of yeast and sugar on $20 \mathrm{mls}$ of warm water. The mixture should be homogenous. The petri dishes were labelled as sun, control, SPF 15, SPF 50, cotton and polyester. The labelling is done on the bottom of the petri dishes not on the lid. One $\mathrm{ml}$ of the yeast solution was put on each petri dish and evenly spread on the agar for even distribution of yeast cells. The yeast solution was allowed to drain into the agar. The inoculation of yeast was done on a sun free environment. The control petri dish was incubated directly while the SPF 15 petri dish top was smeared with sunscreen with SPF 15 and the same one to SPF 50 with the sunscreen with SPF 50. The petri dish labelled with cotton was covered with the cotton cloth and the one labelled with polyester was covered with the polyester cloth. Then all of the petri dishes including the one labelled sun were taken outside to bask on direct sunlight for 6 hours after which the sunscreen was wiped and the clothes removes from the petri dish and then they were incubated for two to three days.

\section{Results and Discussion}

\subsection{Results}

The methods and material mentioned in the methodology section were used in a combination for carrying out the experiment of the current study. After the treat- 
ment of all of these plates according to the defined methods, the results of the study are noted which are presented below.

In the control petri dish there were around 75 countable colonies, in the petri dish labelled sun had no countable colonies, in the SPF 15 there were 30 colonies whereas in SPF 50 there were 60 colonies, in the Cotton petri dish there were 10 colonies whereas in the nylon there were 25 colonies (Table 1 ).

Table 1. Number of colonies in reference to the condition.

\begin{tabular}{|c|c|c|}
\hline No. & Conditions & Colonial growths \\
\hline \multirow{3}{*}{1} & \multirow{3}{*}{$\begin{array}{l}\text { The control petri dish had around } 75 \\
\text { countable colonies. It marked the } \\
\text { optimum growth as compared to the } \\
\text { other petri dishes }\end{array}$} & 75 colonies \\
\hline & & \\
\hline & & 60 colonies \\
\hline 2 & $\begin{array}{l}\text { The petri dish whose lid was covered } \\
\text { with sun screen } 50\end{array}$ & $x^{-\infty}+\infty$ \\
\hline
\end{tabular}

The petri dish that was covered with a cotton cloth
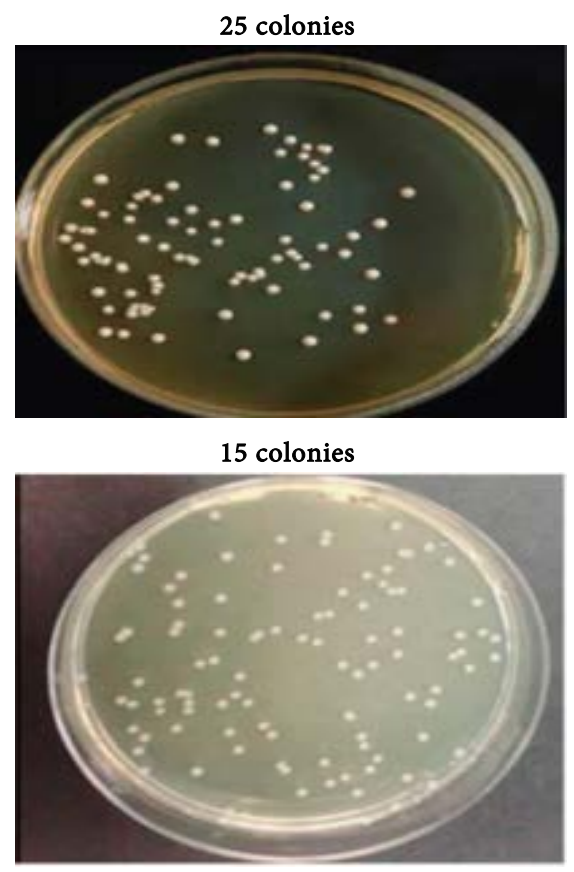


\section{Continued}

The petri dish that was protected with the sun screen SPF 15

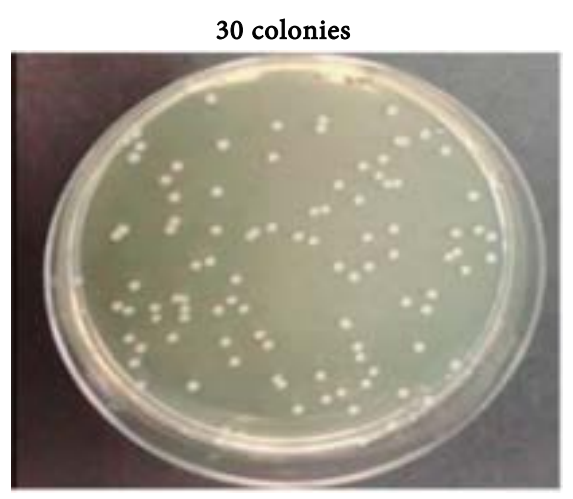

No colonies

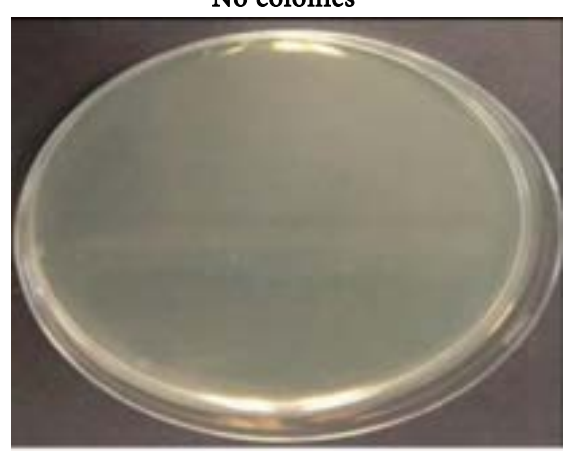
sun petri dish had no countable colonies

\subsection{Analysis and Discussion}

Comparing the growth of yeast cells in the petri dishes it is evident that the petri dish which no sun was exposed has the highest growth recording 75 colonies. This is what we expected from the results since the Petri dish after inoculation was not exposed to sunlight and therefore the yeast cells were not killed and therefore were capable to grow in large numbers. In the petri dish labelled sun it recorded the least growth and infact had no colony growth on it. This is because the UV radiation after being exposed to the sun damaged the repair DNA in the yeast cells and therefore led to the death of almost all the cells due to the mutation they could have undergone. The petri dish labelled PSF 15 had less growth as compared to the petri dish labelled PSF 50; this can be experienced by the life of a sunscreen in protecting photo-radiation. The PSF 50 sunscreen lasted longer and therefore was capable to protect the yeast cell for a longer time that the PSF 15 could do. In comparison between the Petri dish covered with a polyester and cotton, the petri dish covered with polyester recorded a higher growth than the one covered with cotton. This is because industrial material has less space between the fibres as compared to cotton whose fibres are apart allowed the UV rays past it killing more yeast cells (Figure 1).

From the results, the yeast cells need to be shielded from sunlight since it has disastrous effect on the yeast cells; this is the same situation in human cells since have the same effect. The more someone is exposed to the UV radiation the more they are likely to experience heart burns, skin cancers and in the worst case 
Colonial Growth of Yeast in Different Conditions

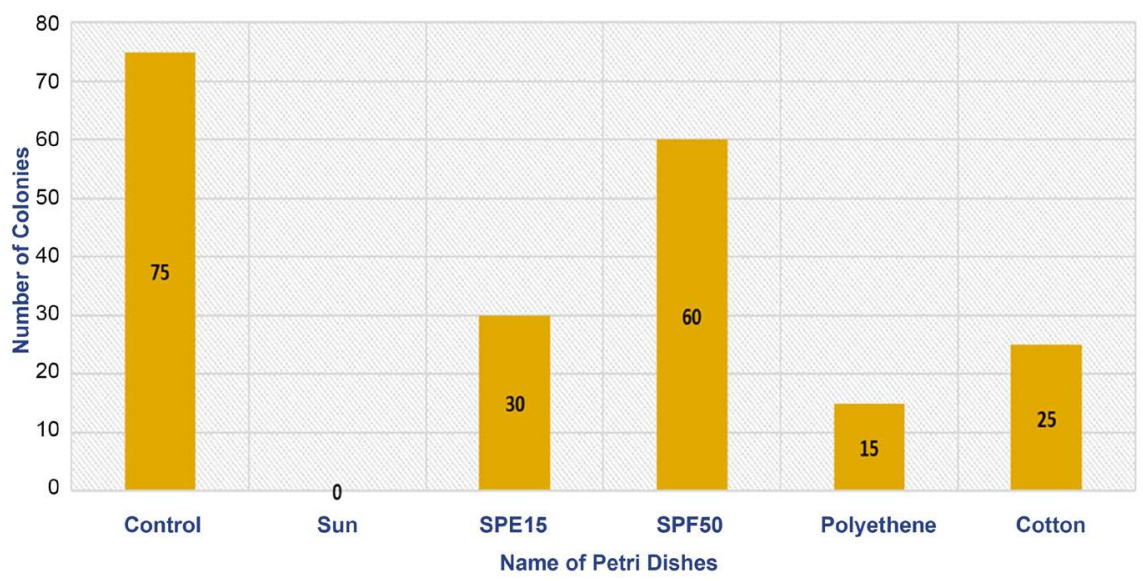

Figure 1. Colonial growth of yeast.

low immunity due to the UVA radiations. Prevention is always better than cure and although someone cannot manage to prevent $100 \%$ of the UV radiation there are various ways to prevent the toxicity of the radiation.

Proper knowledge of the sunscreens can help to curb the UV effects; a sunscreen with a higher PSF value will protect an individual for a longer time than a sunscreen with a lower PSF value. As in the experiment the longer the yeast petri dishes were left in the sun for a long time and the PSF 50 shielded the yeast cells from the radiation for a longer time.

Clothes also have a huge part in curbing UV radiation. Polyester and nylon clothes have a higher UV insulation as compared to cotton clothes. The fabric making cotton is more spread and has more spaces that the industrial made fabric which has less spaces and therefore UV penetrates easily to the skin.

\section{Conclusions}

The findings of the study showed that exposure of the sunlight is necessary for maintaining higher level yeast growth as the plates which were not exposed to sunlight showed relatively lower growth/ml of the farm as compared to the other plates which were exposed to sun with foil or tin plate covers and sunscreens. The sunscreens showed their effectiveness up to $50 \%$ of concentration with and without foil or tin coverings; however, further increase in the concentration of sunscreens backfired as no yeast colonies were witnessed in such cases. The sensitivity of yeast to UV was considered for keeping some plates in incubators and was expected to show the highest growth. However, the growth was the second-lowest after direct exposure with $100 \%$ concentrated sunscreen.

\subsection{Recommendations}

On the basis of conclusions, it is recommended that the combination of coverings for avoiding the extent of exposure and up to $50 \%$ or $30 \%$ concentrated sunscreen can be used for optimal results. In the absence of covering or in the 
case of direct exposure, sunscreen can be used with a $50 \%$ concentration. It is also recommended to avoid using foil or tin covering solely in the case of sun exposure as it may not be able to facilitate the optimal growth of yeast colonies.

\subsection{Future Directions}

The current study remained limited to the analysis of the effects of sunscreens on yeast colonial growth without controlling SPF of sunscreen. There may be an impact of SPF factor as well on the protection of yeast from UV damages. For instance, a higher SPF may be able to yield some colonial growth rather than zero growth. Therefore, it is a future direction for the upcoming studies to consider the application of different sunscreens having different SPF for identifying the exact impact on the yeast growth.

\section{Conflicts of Interest}

The authors declare no conflicts of interest regarding the publication of this paper.

\section{References}

[1] Barahona, S., Yuivar, Y., Socias, G., Alcaíno, J., Cifuentes, V. and Baeza, M. (2016) Identification and Characterization of Yeasts Isolated from Sedimentary Rocks of Union Glacier at the Antarctica. Extremophiles, 4, 479-491. https://doi.org/10.1007/s00792-016-0838-6

[2] Mesías, L.G.G., Qwisgaard, A.M.R., Untivero, G.P.C., Kobayashi, L.A.P., Shimabukuro, L.E.M. and Sugahara, A.A.K. (2017) Comparison of the Photoprotective Effects of Sunscreens Using Spectrophotometric Measurements or the Survivability of Yeast Cells Exposed to UV Radiation. Revista de la Sociedad Química del Perú, 83, 294-307. https://doi.org/10.37761/rsqp.v83i3.113

[3] Shawn Xu, X.Z. and Liu, J.F. (2019) Compositions and Methods for Blocking Ultraviolet Radiation. Michigan University, USA Patent US 2019/0336430 A1, 7 Nov. 2019.

[4] Rosenthal, L. and Burchum, J. (2017) Lehne's Pharmacotherapeutics for Advanced Practice Providers. Elsevier, Amsterdam.

[5] Sudhahar, V. and Balasubramanian, V. (2013) Sun Production Factor (SPF) Determination of Marketed Sunscreen Formulation by In-Vitro Method Using UV-VIS Spectrophotometer. Archives of Applied Science Research, 5, 119-122.

[6] Green, A.C., Williams, G.M., Logan, V. and Strutton, G.M. (2011) Reduced Melanoma after Regular Sunscreen Use: Randomized Trial Follow-Up. Journal of Clinical Oncology, 29, 257-263. https://doi.org/10.1200/JCO.2010.28.7078

[7] Ghiasvand, R., Weiderpass, E., Green, A.C., Lund, E. and Veierød, M.B. (2016) Sunscreen Use and Subsequent Melanoma Risk: A Population-Based Cohort Study. Journal of Clinical Oncology, 34, 3976-3983. https://doi.org/10.1200/JCO.2016.67.5934

[8] Stiefel, C. and Schwack, W. (2015) Photoprotection in Changing Times-UV Filter Efficacy and Safety, Sensitization Processes and Regulatory Aspects. International Journal of Cosmetic Science, 37, 2-30. https://doi.org/10.1111/ics.12165

[9] Waldman, R.A. and Grant-Kels, J.M. (2019) The Role of Sunscreen in the Preven- 
tion of Cutaneous Melanoma and Nonmelanoma Skin Cancer. Journal of the American Academy of Dermatology, 80, 574-576. https://doi.org/10.1016/j.jaad.2018.06.069

[10] Shaath, N.A. (2010) Ultraviolet Filters. Photochemical \& Photobiological Sciences, No. 4, 405-616. https://doi.org/10.1039/b9pp00174c

[11] Kowalski, W. (2010) Ultraviolet Germicidal Irradiation Handbook: UVGI for Air and Surface Disinfection, Madison Ave. Springer Science \& Business Media, New York. https://doi.org/10.1007/978-3-642-01999-9

[12] Goodman, C.C. and Fuller, K.S. (2014) Pathology: Implications for the Physical Therapist. 4th Edition, Elsevier Health Sciences, Amsterdam, 1800.

[13] Jansen, R., Osterwalder, U., Wang, S.Q., Burnett, M. and Lim, H.W. (2013) Photoprotection: Part II. Sunscreen: Development, Efficacy, and Controversies. Journal of the American Academy of Dermatology, 69, 867-e1. https://doi.org/10.1016/j.jaad.2013.08.022

[14] Hacker, E., Horsham, C., Ford, H., Hartel, G., Olsen, C.M., Pandeya, N. and Janda, M. (2019) UV Detection Stickers Can Assist People to Reapply Sunscreen. Preventive Medicine, 124, 67-74. https://doi.org/10.1016/j.ypmed.2019.05.005

[15] Mazzarello, V., Ferrari, M., Piu, G., Pomponi, V. and Solinas, G. (2019) Do Sunscreen Prevent Recurrent Herpes Labialis in Summer? Journal of Dermatological Treatment, 30, 179-182. https://doi.org/10.1080/09546634.2018.1481921

[16] Petersen, B. and Wulf, H.C. (2014) Application of Sunscreen-Theory and Reality. Photodermatology, Photoimmunology \& Photomedicine, 30, 96-101. https://doi.org/10.1111/phpp.12099

[17] Smaoui, S., Hlima, H.B., Chobba, I.B. and Kadri, A. (2017) Development and Stability Studies of Sunscreen Cream Formulations Containing Three Photo-Protective Filters. Arabian Journal of Chemistry, 10, S1216-S1222. https://doi.org/10.1016/j.arabjc.2013.02.020

[18] da Silva, A.C.P., Paiva, J.P., Diniz, R.R., Dos Anjos, V.M., Silva, A.B.S.M., Pinto, A.V., Dos Santos, E.P., et al. (2019) Photoprotection Assessment of Olive (Olea europaea L.) Leaves Extract Standardized to Oleuropein: In Vitro and in Silico Approach for Improved Sunscreens. Journal of Photochemistry and Photobiology B: Biology, 193, 162-171. https://doi.org/10.1016/j.jphotobiol.2019.03.003

[19] Bereketoğlu, C. (2017) Investigation of the Effects of Bisphenol A and Nonylphenol on the Transcriptional Profiles of Saccharomyces Cerevisiae Genome. Marmara University, Istanbul.

[20] Yang, G., Cozad, M.A., Holland, D.A., Zhang, Y., Luesch, H. and Ding, Y.S. (2018) Photosynthetic Production of Sunscreen Shinorine Using an Engineered Cyanobacterium. ACS Synthetic Biology, 7, 664-671. https://doi.org/10.1021/acssynbio.7b00397

[21] Kumar, D., Karthik, L., Kumar, G. and Roa, K.B. (2011) Biosynthesis of Silver Anoparticles from Marine Yeast and Their Antimicrobial Activity against Multidrug Resistant Pathogens. Pharmacologyonline, 3, 1100-1111.

[22] Libkind, D., Moliné, M., Trochine, A., Bellora, N. and de Garcia, V. (2016) Biotechnologically Relevant Yeasts from Patagonian Natural Environments. In: Biology and Biotechnology of Patagonian Microorganisms, Springer, Cham, 325-351. https://doi.org/10.1007/978-3-319-42801-7_18

[23] Marrot, L., Labarussiat, A., Perez, P. and Meunier, J.R. (2006) Use of the Yeast Saccharomyces cerevisiae as a Pre-Screening Approach for Assessment of Chemical-Induced Phototoxicity. Toxicology in Vitro, 20, 1040-1050. 
https://doi.org/10.1016/j.tiv.2006.01.008

[24] Kim, S. and Choi, K. (2014) Occurrences, Toxicities, and Ecological Risks of Benzophenone-3, a Common Component of Organic Sunscreen Products: A Mini-Review. Environment International, 1, 143-157. https://doi.org/10.1016/j.envint.2014.05.015

[25] Geoffrey, K., Mwangi, A.N. and Maru, S.M. (2019) Sunscreen Products: Rationale for Use, Formulation Development and Regulatory Considerations. Saudi Pharmaceutical Journal: SP): The Official Publication of the Saudi Pharmaceutical Society, 27, 1009-1018. https://doi.org/10.1016/j.jsps.2019.08.003

[26] Diniz, R.R., Paiva, J.P., Aquino, R.M., Gonçalves, T.C.W., Leitão, A.C., Santos, B.A.M.C., Pinto, A.V., Leandro, K.C. and de Pádula, M. (2019) Saccharomyces cerevisiae Strains as Bioindicators for Titanium Dioxide Sunscreen Photoprotective and Photomutagenic Assessment. Journal of Photochemistry and Photobiology B: Biology, 198, Article ID: 111584. https://doi.org/10.1016/j.jphotobiol.2019.111584 